CARDIOVASCULAR MEDICINE

\title{
Early invasive strategy in elderly patients with non- ST elevation acute coronary syndrome: comparison with younger patients regarding 30 day and long term outcome
}

\author{
F Liistro, P Angioli, G Falsini, K Ducci, S Baldassarre, A Burali, L Bolognese
}

Heart 2005;91:1284-1288. doi: 10.1136/hrt.2004.051607

See end of article for authors' affiliations

Correspondence to: Dr Francesco Liistro, Department of Cardiovascular Disease San Donato Hospital 52100 Arezzo, Italy; francescoliistro@hotmail. com

Accepted 1 December 2004 Published Online First 10 March 2005

\begin{abstract}
Objectives: To evaluate an early invasive strategy in elderly patients with non-ST elevation acute coronary syndrome (ACS).

Methods: All consecutive patients admitted with a diagnosis of non-ST elevation ACS from June 2002 to February 2004 were enrolled in this registry. Clinical outcome was assessed at 30 days and in long term follow up.

Results: An early invasive strategy was followed for 439 patients, of whom 159 (36\%) were elderly and had a higher clinical risk profile and greater extent of coronary artery disease (CAD) than the younger patients. Coronary revascularisation was conducted in 133 (83\%) elderly patients and 239 (85\%) younger patients (not significant). At a mean (SD) follow up time of 10.7 (5.2) months overall mortality, cardiac death, and death plus myocardial infarction were significantly higher among elderly patients than among younger patients $19.4 \% \vee 2.1 \%, p<0.001 ; 6.8 \% \vee 1.8 \%, p<0.01 ; 11.3 \% \vee 5 \%, p=0.02$, respectively). The significant difference in cardiac death between the two groups was related more to elderly patients being treated by coronary artery bypass grafting (19.3\% v 4.9\%, $p=0.05)$ than by percutaneous coronary intervention $(\mathrm{PCl})(2.9 \%$ v $1.1 \%, \mathrm{p}=0.3)$. Cox regression analysis showed age, serum creatinine $>115 \mu \mathrm{mol} / \mathrm{l}$, no previous history of $C A D$, left ventricular ejection fraction $>45 \%$, and the absence of diabetes to be independent predictors of the occurrence of major adverse cardiac events. Conclusions: In unselected elderly patients presenting with non-ST elevation ACS an early invasive strategy is feasible and leads to coronary revascularisation in the majority of cases, resulting in encouraging immediate and long term clinical results, particularly among $\mathrm{PCl}$ treated patients.
\end{abstract}

$\mathrm{R}$ ecent randomised trials ${ }^{1-4}$ have shown improved clinical outcomes among high risk patients with non-ST elevation acute coronary syndrome (ACS) with an early invasive strategy including routine use of diagnostic catheterisation and revascularisation as indicated. Apart from the fact that these recent studies have been appropriately powered to determine potential differences between treatment strategies, a critical component of these studies has been the reluctance to enrol elderly patients, particularly those with co-morbid conditions. ${ }^{5}$ The elderly constitute an increasing proportion of patients presenting with non-ST elevation ACS and are at increased risk because of the greater extent of coronary artery disease (CAD) and associated comorbid conditions. The uncertainty about treatment strategies is further increased by the limited available information about the outcome of such patients undergoing different treatment modalities. In a recently published registry of patients with non-ST elevation ACS, ${ }^{6}$ over $35 \%$ of the patients were over the age of 75 years, emphasising the growing importance of examining outcomes in the older age group. Furthermore, a significant difference was found in the treatment modalities between elderly patients (invasive strategy in $39 \%$ ) and younger patients (invasive strategy in $56 \%$ ) with a 30 day mortality among elderly patients nearly fourfold as high as that of the younger population. On the other hand, a recently published post hoc retrospective analysis of the TACTICS-TIMI (treat angina with Aggrastat and determine cost of therapy with an invasive or conservative strategy-thrombolysis in myocardial infarction) 18 study showed that an early invasive strategy can significantly improve outcome among elderly patients with non-ST elevation ACS-compelling evidence to support a shift towards an aggressive treatment strategy. ${ }^{7}$ Thus, we investigated early and late clinical outcome in a consecutive series of patients presenting with non-ST elevation ACS and systematically treated with an early invasive strategy, outlining the differences between elderly and younger patients.

\section{METHODS}

Patients and study protocol

The study population consisted of 463 patients with non-ST elevation ACS consecutively referred to our coronary care unit from June 2002 to February 2004. They were prospectively enrolled in this study if they met at least two of the following inclusion criteria: (1) angina at rest lasting $>10$ minutes within 12 hours of hospital admission; (2) unequivocal changes (transient or persistent pathological ST segment depression of $0.1 \mathrm{mV}$ or $\mathrm{T}$ wave inversion in at least two adjacent leads without pathological Q waves) on ECG during angina; and (3) cardiac troponin I (cTnI) rise. Exclusion criteria were (a) inability to provide informed consent; (b) concomitant non-cardiac life threatening disease; and (c)

Abbreviations: ACS, acute coronary syndrome; $C A B G$, coronary artery bypass grafting; $C A D$, coronary artery disease; $C l$, confidence interval; CK, creatine kinase; cTnl, cardiac troponin I; LVEF, left ventricular ejection fraction; MACE, major adverse cardiac events; $O R$, odds ratio; $\mathrm{PCl}$, percutaneous coronary intervention; TACTICS-TIMI, treat angina with Aggrastat and determine cost of therapy with an invasive or conservative strategy-thrombolysis in myocardial infarction 
severe haemodynamic impairment or cardiogenic shock (hypotension with systolic blood pressure $<90 \mathrm{~mm} \mathrm{Hg}$ and heart rate $>100$ beats/min, not caused by hypovolaemia and requiring inotropic support or balloon counterpulsation); we applied no upper age limit.

Of the 463 patients initially selected for the study, 24 (5\%) were lost to the follow up protocol. Thus, 439 patients (303 men; mean (SD) age 68.1 (11.2) years, range 30-92 years) constituted the final study group. The research protocol was approved by the hospital ethics committee, and one of the investigators obtained informed consent from each patient.

All patients were required to undergo coronary angiography within 24-48 hours of admission. Blood samples for determination of CTnI, creatine kinase (CK), and CK-MB concentrations were obtained on admission and every six hours thereafter up to 48 hours and at 6, 12, 18, and 24 hours after percutaneous coronary intervention (PCI), if performed.

Myocardial revascularisation and concomitant drugs PCI and stent implantation were performed according to institutional standards. Single vessel disease was defined as the presence of a single or multiple stenoses $>50 \%$ in a single coronary vessel. Multivessel disease was defined as the presence of single or multiple stenoses $>50 \%$ in more than one coronary vessel. In patients with single vessel disease, the culprit lesion was considered to be the most severely stenosed lesion in the affected vessel. In patients with multivessel disease, the culprit lesion was defined as a substantially stenosed lesion in the vessel that corresponded most closely to the ischaemic area as determined by ST-T segment changes during chest pain. Heparin was given intravenously to achieve an activated clotting time of 250-300 seconds or $\geqslant 200$ seconds if the patient was taking glycoprotein IIb/IIIa receptor antagonists. Postprocedural heparin infusion was continued for 12 hours. All patients received aspirin (100$300 \mathrm{mg}$ ) before and after PCI. Ticlopidine $(500 \mathrm{mg})$ or clopidogrel (loading dose $300 \mathrm{mg}$ followed by $75 \mathrm{mg} /$ day) was administered before PCI and daily thereafter for at least 30 days. Upstream or downstream glycoprotein IIb/IIIa was given according to the house physician's and operator's preference.

The indication for surgical or percutaneous myocardial revascularisation was based on the extent of CAD and the patient's surgical risk profile. ${ }^{8}$ Patients with multivessel CAD were considered candidates for coronary artery bypass grafting (CABG) in the following cases: (1) acceptable surgical risk; (2) left main disease; (3) multivessel disease with long and diffuse lesions; (4) multivessel disease with more than one chronic total occlusion of a major vessel; and (5) multivessel disease and concomitant moderate to severe ischaemic mitral insufficiency. In patients with multivessel disease for whom PCI was considered suitable, an attempt was made to achieve complete myocardial revascularisation if clinically indicated.

PCI was performed in the same session as diagnostic angiography. CABG was performed within a week after diagnostic angiography. Treatment with glycoprotein IIb/IIIa and platelet ADP receptor antagonists was discontinued immediately after diagnostic coronary angiography.

\section{Definitions and outcome measures}

Successful PTCA was defined as the restoration of TIMI 3 grade flow and residual stenosis $\leqslant 30 \%$ at the end of the procedure. Major adverse cardiac events (MACE) were defined as death, non-fatal reinfarction, non-fatal stroke, and repeat revascularisation. All deaths were considered to be of cardiac origin unless a non-cardiac origin was established clinically or at necropsy. Reinfarction was diagnosed as follows: (1) an increase of CK or its isoform to at least twice
Table 1 Baseline clinical characteristics

\begin{tabular}{llll}
\hline & $\begin{array}{l}\text { Age }<\mathbf{7 5} \\
(\mathbf{n = 2 8 0 )}\end{array}$ & $\begin{array}{l}\text { Age } \geqslant \mathbf{7 5} \\
(\mathbf{n = 1 5 9 )}\end{array}$ & p Value \\
\hline Men & $224(80 \%)$ & $79(49.6 \%)$ & $<0.0001$ \\
Family history of CAD & $40(14 \%)$ & $2(1.2 \%)$ & $<0.0001$ \\
Smoking & $44(15.7 \%)$ & $2(1.2 . \%)$ & $<0.001$ \\
Hypertension & $104(37.1 \%)$ & $64(40.2 \%)$ & NS \\
Diabetes & $57(20.3 \%)$ & $32(20.1 \%)$ & NS \\
Hypercholesterolaemia & $70(25 \%)$ & $22(13.8 \%)$ & $<0.01$ \\
History of CAD & $78(27.8 \%)$ & $35(22.0 \%)$ & NS \\
Previous myocardial & $38(13.6 \%)$ & $13(8.2 \%)$ & NS \\
revascularisation & $11(3.9 \%)$ & $13(8.1 \%)$ & 0.07 \\
Carotid artery disease & $7(2.5 \%)$ & $7(4.4 \%)$ & NS \\
Peripheral artery disease & $1.0(0.6)$ & $1.2(0.3)$ & 0.05 \\
Serum creatinine (mg/ml) & $55.2(7.6)$ & $50.4(7.3)$ & 0.02 \\
LVEF (\%) & $210(75 \%)$ & $138(87 \%)$ & 0.01 \\
ECG changes & $146(52 \%)$ & $99(62 \%)$ & 0.04 \\
cTnl rise & $106(37.8 \%)$ & $45(28.3 \%)$ & 0.04 \\
GP Ilb/Illa inhibitors & & & \\
\hline
\end{tabular}

Data are number $(\%)$ or mean (SD).

$\mathrm{CAD}$, coronary artery disease; $\mathrm{CTnl}$, cardiac troponin I; GP, glycoprotein; LVEF, left ventricular ejection fraction; NS, not significant.

the upper limit of the normal reference range; (2) an increase of CK or its isoform to three times the upper limit of the normal value within 48 hours after PCI or to five times the upper limit after $\mathrm{CABG}^{9}$; or (3) development of a new Q wave on the ECG in at least two contiguous leads. For purposes of survival analyses, only one event (the first that occurred) was tabulated for each patient. After hospital discharge, patients were referred to their private physician, who regulated treatment. No attempt was made to standardise treatment. All patients were asked to return to our outpatient clinic for evaluation by one of the investigators six months after discharge and annually thereafter. For those patients who did not return to the clinic at the designated time, follow up information was collected by telephone interview.

\section{Statistical analysis}

Continuous data are expressed as mean (SD). Baseline data were compared by means of the Fisher's exact test for categorical variables and unpaired $t$ test for normally distributed continuous variables. Kaplan-Meier survival analysis was used to assess event-free survival at 12 months. The multivariate Cox proportional hazards regression model was used to identify independent predictors of long term mortality and MACE. Clinical, demographic, and angiographic variables that were significantly different between survivors and non-survivors in the univariate model (age, diabetes, left main stenosis, history of CAD, left ventricle ejection fraction (LVEF), serum creatinine concentration), as well as those known to have prognostic value (cTnI rise, sex,

Table 2 Angiography and myocardial revascularisation

\begin{tabular}{llll}
\hline & $\begin{array}{l}\text { Age }<75 \\
(\mathbf{n = 2 8 0 )}\end{array}$ & $\begin{array}{l}\text { Age } \geqslant 75 \\
(\mathbf{n = 1 5 9 )}\end{array}$ & p Value \\
\hline CAD & $20(7 \%)$ & $9(5 \%)$ & NS \\
No CAD & $130(46 \%)$ & $58(36 \%)$ & 0.04 \\
Single vessel disease & $134(48 \%)$ & $92(59 \%)$ & 0.04 \\
Multivessel disease & $39(13 \%)$ & $28(17 \%)$ & NS \\
Left main stenosis & $239(85 \%)$ & $133(83 \%)$ & NS \\
Myocardial revascularisation & $41(15 \%)$ & $26(16.3 \%)$ & NS \\
No revascularisation performed & $178(63 \%)$ & $102(64.4 \%)$ & NS \\
PCl & $70(25 \%)$ & $32(20.3 \%)$ & NS \\
Multivessel intervention & $61(22 \%)$ & $31(19 \%)$ & NS \\
CABG & & & \\
\hline
\end{tabular}

Data are number (\%).

$\mathrm{CABG}$, coronary artery bypass grafting; $\mathrm{PCl}$, percutaneous coronary intervention. 
Table 3 Clinical and angiographic variables in patients undergoing $\mathrm{PCl}$ and $\mathrm{CABG}$

\begin{tabular}{llll}
\hline & PCI $(\mathbf{n = 2 8 0 )}$ & CABG (n=92) & p Value \\
\hline Age (years) & $67.9(11.7)$ & $68.2(10.0)$ & NS \\
Men & $204(72.8 \%)$ & $61(66.3 \%)$ & NS \\
Diabetes & $68(24.2 \%)$ & $15(16.3 \%)$ & NS \\
Carotid artery disease & $20(7.1 \%)$ & $4(4.3 \%)$ & NS \\
Peripheral artery disease & $13(4.6 \%)$ & $1(1.1 \%)$ & NS \\
Serum creatinine (mg/ml) & $1.1(0.7)$ & $1.1(0.20$ & NS \\
LVEF (\%) & $54(5.3)$ & $46.2(11.3)$ & $<0.001$ \\
Multivessel disease & $139(46.5 \%)$ & $77(83.6 \%)$ & $<0.0001$ \\
Left main stenosis & $14(5 \%)$ & $46(50 \%)$ & $<0.0001$ \\
\hline \multirow{2}{*}{ Data are number (\%) or mean (SD). } & & \\
\hline
\end{tabular}

the use of IIb/IIIa receptor antagonists, and multivessel disease), were included in the Cox model. A probability value of $p \leqslant 0.05$ was considered significant. Statistical analysis was performed with SPSS 8.0 for Windows (SPSS Inc, Chicago, Illinois, USA).

\section{RESULTS}

\section{Baseline patient characteristics}

There were 159 (36\%) patients over the age of 75 years (mean age $79.2(4.1)$ years) and $280(64 \%)$ aged $<75$ years (mean age 61.9 (8.7) years). Table 1 reports the baseline clinical characteristics of the two groups. Elderly patients were more likely to be women with a lower incidence of hypercholesterolaemia, ${ }^{10}$ smoking habit, ${ }^{11}$ and family history of CAD. ${ }^{12}$ Certain characteristics traditionally associated with higher risk among patients with ACS, including lower LVEF, higher serum creatinine, increased cTnI concentrations, and ECG changes, were more common among elderly patients However, despite their higher risk profile, fewer elderly than young patients were taking glycoprotein IIb/IIIa inhibitors.

\section{Coronary angiography and revascularisation}

Table 2 reports the angiographic variables and revascularisation rates. There was no difference in lack of angiographic evidence of coronary stenosis between the two groups. However, multivessel disease was more common in the elderly population. Myocardial revascularisation was performed in 133 (83\%) elderly patients and in 239 (85\%) (not significant) younger patients with no difference between the groups in the technique, percutaneous or surgical, that was applied. Table 3 reports the clinical and angiographic characteristics of patients undergoing PCI and CABG. CABG

\begin{tabular}{llll} 
Table 4 & Early and late clinical outcome & \\
\hline & $\begin{array}{l}\text { Age }<\mathbf{7 5} \\
(\mathbf{n = 2 8 0 )}\end{array}$ & $\begin{array}{l}\text { Age } \geqslant \mathbf{7 5} \\
(\mathbf{n}=159)\end{array}$ & $\mathrm{p}$ Value \\
\hline 30 day mortality & $1(0.3 \%)$ & $5(3.1 \%)$ & 0.02 \\
PCl & 0 & $2(1.9 \%)$ & $\mathrm{NS}$ \\
CABG & $1(1.6 \%)$ & $2(6.4 \%)$ & $\mathrm{NS}$ \\
30 day death + non-fatal MI & $3(1 \%)$ & $9(5.6 \%)$ & 0.01 \\
Long term outcome & & & \\
Overall mortality & $6(2.1 \%)$ & $15(9.4 \%)$ & $<0.001$ \\
Death + non-fatal MI & $14(5 \%)$ & $18(11.3 \%)$ & 0.02 \\
Cardiac death & $5(1.8 \%)$ & $11(6.8 \%)$ & $<0.01$ \\
PCI & $1.1 \%$ & $2.9 \%$ & $\mathrm{NS}$ \\
CABG & $4.9 \%$ & $19.3 \%$ & 0.05 \\
MI & $8(2.8 \%)$ & $5(3.1 \%)$ & $\mathrm{NS}$ \\
Repeat revascularisation & $21(7.5 \%)$ & $15(9.4 \%)$ & $\mathrm{NS}$ \\
PTCA & $6(2.1 \%)$ & $2(1.2 \%)$ & $\mathrm{NS}$ \\
CABG & $2(0.7 \%)$ & $2(1.2)$ & $\mathrm{NS}$ \\
Stroke & $244(87.1 \%)$ & $128(80.5 \%)$ & 0.07 \\
Event-free survival & & & \\
\hline Data are number (\%). & & & \\
MI, myocardial infarction. & & &
\end{tabular}

was indicated because of left main stenosis $>60 \%$ in 16 $(10 \%)$ elderly patients and in 32 ( $11 \%)$ (not significant) younger patients and for the presence of three vessel disease with multiple lesions or chronic total occlusions in $12(7 \%)$ elderly patients and $29(10 \%)$ (not significant) younger patients. Patients undergoing CABG had more severe coronary disease and a lower LVEF. Logistic Euroscore was 4.9 (4) in younger patients and 13.2 (9) in elderly patients $(\mathrm{p}<0.001)$.

\section{Early and late clinical outcome}

The mean (SD) duration of clinical follow up was 10.7 (5.2) months (range 6-24 months). During this period, 13 (3\%) patients had a non-fatal reinfarction, $4(0.9 \%)$ had a disabling stroke, and 21 (4.8\%) died. Additional revascularisation procedures were performed in $44(10 \%)$ patients (table 4).

At 30 days, death and death plus non-fatal myocardial infarction were significantly more common among elderly than among younger patients $(3.1 \% \vee 0.3 \%, \mathrm{p}=0.02$ and $5.6 \% v 1 \%, \mathrm{p}=0.01$, respectively). A trend towards a higher mortality was observed among patients who underwent CABG than among those who underwent PCI $(3.2 \% v 0.7 \%$, $p=0.09$ ) and among elderly than among younger patients in each treatment modality $(6.4 \% v 1.6 \%, \mathrm{p}=0.2$ for $\mathrm{CABG}$ and $1.9 \% \vee 0 \%$ for PCI, $\mathrm{p}=0.1$ ).

At long term follow up, overall mortality, cardiac death, and death plus non-fatal myocardial infarction were significantly more common among elderly than among younger patients $(9.4 \% \vee 2.1 \%, \mathrm{p}<0.001 ; 6.8 \% \vee 1.8 \%, \mathrm{p}<0.01$; $11.3 \% \vee 5 \%, p=0.02$, respectively). However, the significant difference in cardiac death between the two groups was related more to patients treated by CABG (19.3\% $v 4.9 \%$, $\mathrm{p}=0.05)$ than to those treated by PCI $(2.9 \% \vee 1.1 \%$, $\mathrm{p}=0.3)$. The difference in cardiac mortality between elderly patients treated by CABG and those treated by PCI $(19.3 \% v$ $2.9 \%, \mathrm{p}<0.01)$ became not significant after adjustment for LVEF.

Event-free survival was $80.5 \%$ in elderly patients versus $87.1 \%$ in younger patients $(p=0.07)$. Figure 1 reports the 12 month Kaplan-Meier event-free survival.

Multivariate regression analysis by the Cox model for the entire population showed age (odds ratio (OR) 1.09, 95\% confidence interval (CI) 1.02 to 1.15 ), serum creatinine $>115 \mu \mathrm{mol} / \mathrm{l}$ (OR 1.6, 95\% CI 1.18 to 2.24 ), the absence of left main disease (OR $0.26,95 \%$ CI 0.10 to 0.66 ), the absence of a history of CAD (OR $0.30,95 \%$ CI 0.10 to 0.86 ), and LVEF $>45 \%$ (OR $0.91,95 \%$ CI 0.88 to 0.95 ) to be independent

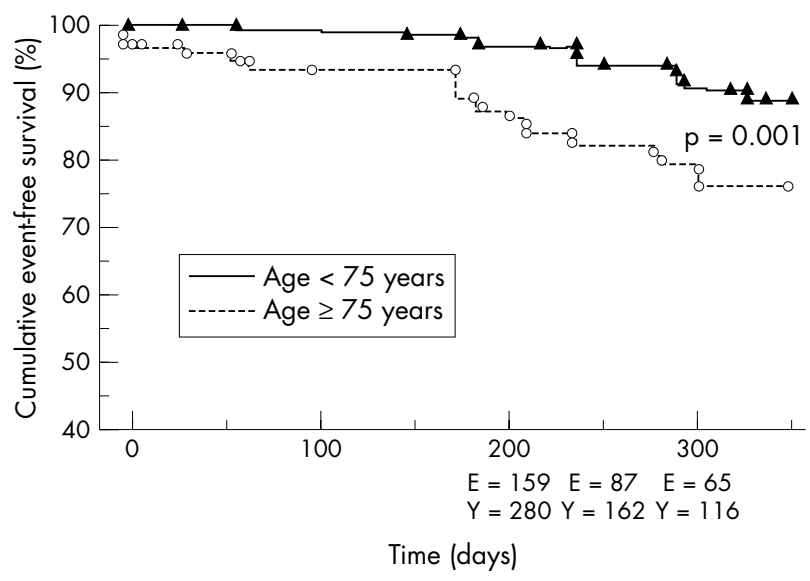

Figure 1 Kaplan-Meier cumulative plot for 12 month event-free survival among elderly $(E)$ and younger $(Y)$ patients who completed follow up. 


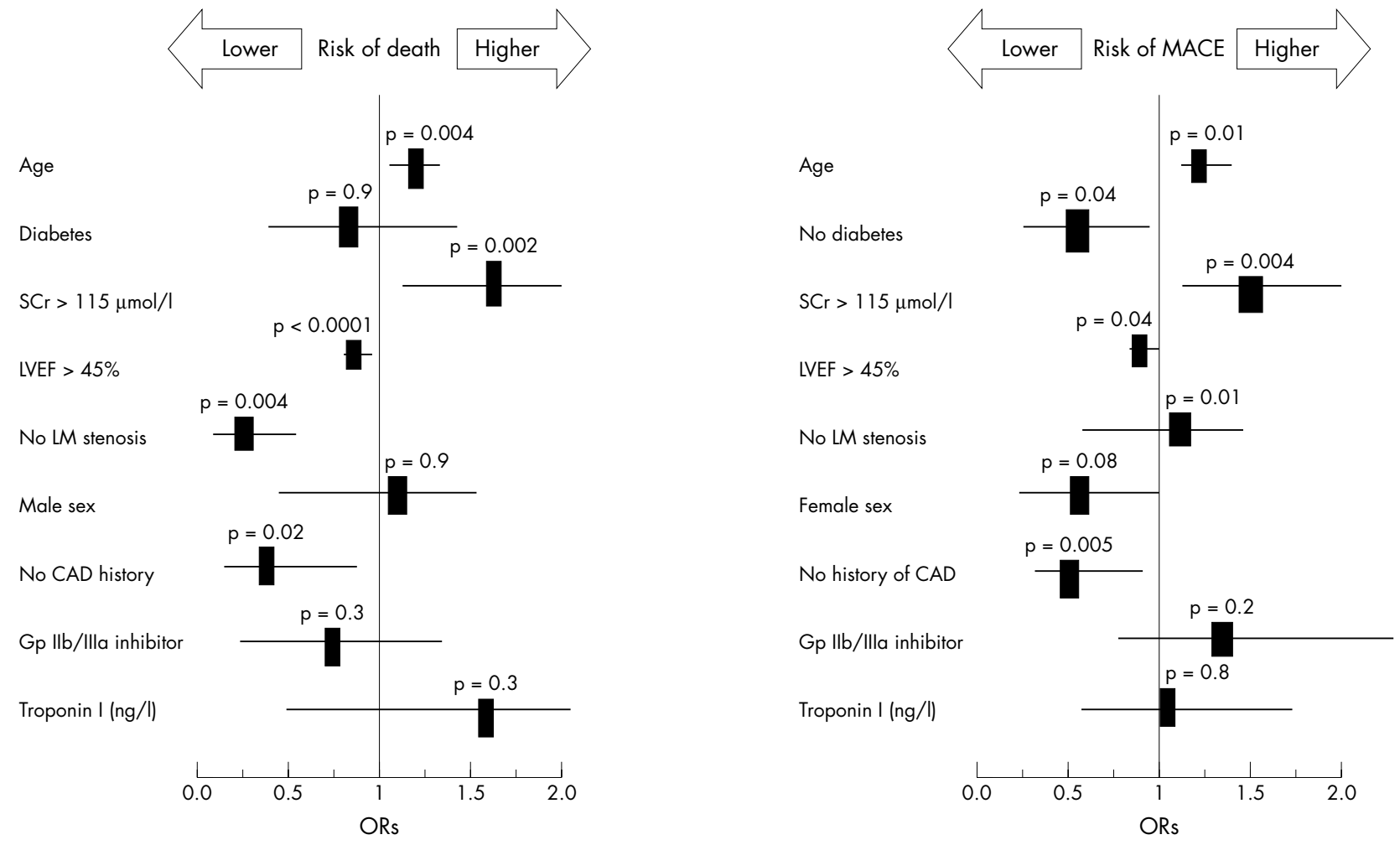

Figure 2 Odds ratios (ORs) and 95\% confidence intervals for death (left) and major adverse cardiac events (MACE; right) during long term follow up of patients with non-ST elevation acute coronary syndrome. CAD, coronary artery disease; Gp, glycoprotein; LM, left main; LVEF, left ventricle ejection fraction; $\mathrm{SCr}$, serum creatinine.

predictors of long term mortality. Age (OR 1.03, 95\% CI 1.00 to 1.06 ), serum creatinine $>115 \mu \mathrm{mol} / \mathrm{l}$ (OR $1.41,95 \%$ CI 1.11 to 1.81 ), the absence of a history of CAD (OR $0.46,95 \%$ CI 0.27 to 0.80 ), $\mathrm{LVEF}>45 \%$ (OR $0.96,95 \%$ CI 0.93 to 0.99 ), and the absence of diabetes (OR $0.54,95 \%$ CI 0.31 to 0.97 ) were independent predictors of long term MACE occurrence (fig 2). No significant difference in MACE occurrence was observed between patients with cTnI rise and those without in the entire population $(84.0 \% \vee 85.3 \%$, respectively, not significant), as well as between elderly $(78.3 \% \vee 81.8 \%$, respectively, not significant) and younger (86.5\% $\vee 87.6 \%$, respectively, not significant) cohorts analysed separately.

\section{DISCUSSION}

As reported in other studies, our results confirm that elderly patients constitute a significant portion (about 30\%) of the total population requiring medical care for non-ST elevation ACS with a worse cardiac risk profile than that of younger patients. ${ }^{13}$ In elderly patients undergoing myocardial revascularisation either by CABG or by PCI, morbidity and mortality are higher than those observed in younger patients. ${ }^{15-17}$ This observation has raised clinicians' thresholds for considering aggressive revascularisation strategies for the elderly with non-ST elevation ACS. Furthermore, early randomised trials focusing on early invasive strategy in patients with ACS excluded the elderly, such that data from younger patients have had to be extrapolated to older age groups in the clinical arena.

The results of the present study indicate that an early invasive strategy, including routine use of diagnostic catheterisation within 24-48 hours of admission and revascularisation as indicated, is feasible for a substantial proportion of elderly patients with non-ST elevation ACS with a reasonable amount of major complications occurring during hospitalisation and long term follow up. In particular in the present study, although results of PCI were worse in elderly than in younger patients, the number of adverse events in elderly patients was acceptable, since 30 day mortality was $1.9 \%$ and long term cardiac mortality was $2.9 \%$, figures that compare favourably with previously reported data in this population. Morrison $e^{2}$ al $^{18}$ reported a 30 day survival of $87 \%$ among 131 patients $\geqslant 70$ years of age treated with angioplasty for unstable angina. In the experience reported by Munoz et al ${ }^{19}$ with 76 patients $\geqslant 75$ years of age, of whom $79 \%$ had unstable angina, hospital mortality was $6.6 \%$. In another registry ${ }^{20}$ of 449 patients with ACS aged $\geqslant 70$ years, an invasive approach was used in $168(37 \%)$ patients and inhospital mortality was $3.1 \%$ in the total population, $0.8 \%$ in patients aged $<80$ years, and $6.1 \%$ in those aged $\geqslant 80$ years. Furthermore, in these registries selection criteria for the invasive approach were not specified, and patients who underwent angiography were indeed at lower risk than were those treated medically. Our data are similar to those reported in a recent published post hoc analysis of the TACTICS-TIMI 18 trial $^{7}$ where the six month death and myocardial infarction rate was $10.8 \%$ in elderly patients treated with an early invasive strategy versus $21.6 \%$ in those treated conservatively $(p=0.01)$. However, in that trial elderly patients with co-morbid conditions were excluded and consequently the results may not be directly transferred to an unselected elderly non-ST elevation ACS patient population. The present study had the advantage of examining an unselected cohort of consecutive patients with non-ST elevation ACS. Since it was a single centre study, it was possible to ensure that all elderly patients with ACS hospitalised within a given time period were included, thus avoiding selection bias, which may be a critical factor in the less exact reporting that may occur in multicentre registries. Lastly, an early invasive strategy with percutaneous or surgical revascularisation was completed in almost the whole 
population with no differences in terms of the technique, surgical or percutaneous, applied in both cohorts.

Mortality among elderly patients undergoing CABG in our study was $6.4 \%$ at 30 days and $19.3 \%$ in the long term. These rates may be explained by the high value of logistic Euroscore $(13 \%)$ of these patients. Similar results were reported from a monocentre study ${ }^{21}$ of 388 octogenarians undergoing isolated CABG with an overall in-hospital mortality of $9.1 \%$ (ranging from $6.7 \%$ in elective procedures to $21 \%$ in emergent procedures) and in a larger multicentre experience ${ }^{22}$ of 4743 octogenarians undergoing CABG with an in-hospital mortality of $8.1 \%$.

In our study MACE occurrence did not differ between patients with and without a cTnI rise. Previous trials ${ }^{23} 24$ showed that among patients with clinically documented ACS, even small increases in cTnI identify high risk patients who benefit significantly from an early invasive strategy. However, all of our patients underwent early coronary angiography and most underwent myocardial revascularisation, independently of cTnI. This observation probably explains the similar MACE occurrence between patients with cTnI rise and those without.

\section{Study limitations}

The main limitations of the study are that it was not a randomised trial capable of providing a completely unbiased assessment of treatment effects. Furthermore, as a single centre study, findings may have been influenced by local patient referral and physician practice patterns and, therefore, need to be confirmed in additional patient cohorts. However, we examined an unselected cohort of consecutive patients with non-ST elevation ACS ensuring that all elderly patients with ACS hospitalised within a given time period were included.

\section{Conclusions}

Data from the present study show that in unselected elderly patients presenting with non-ST elevation ACS an early invasive strategy is feasible and leads to coronary revascularisation in the majority of cases, resulting in encouraging immediate and long term clinical results, particularly among PCI treated patients. The growing prevalence of elderly patients presenting with non-ST elevation ACS prompts further investigations in dedicated randomised clinical trials to assess the appropriate strategy to apply in this setting.

\section{Authors' affiliations \\ F Liistro, P Angioli, G Falsini, K Ducci, S Baldassarre, A Burali, L Bolognese, Department of Cardiovascular Disease, San Donato Hospital, Arezzo, Italy}

\section{REFERENCES}

1 Cannon CP, Weintraub WS, Demopoulos LA, et al. Comparison of early invasive and conservative strategies in patients with unstable coronary syndromes treated with the glycoprotein Illb/llla inhibitor tirofiban. NEngl J Med 2001;344:1879-87

2 FRISC II Investigators. Invasive compared with non-invasive treatment in unstable coronary-artery disease: FRISC II prospective randomised multicentre study. Fragmin and fast revascularisation during instability in coronary artery disease investigators. Lancet 1999;354:708-15.

3 Fox KA, Poole-Wilson PA, Henderson RA, et al. Interventional versus conservative treatment for patients with unstable angina or non-ST-elevation myocardial infarction: the British Heart Foundation RITA 3 randomised trial. Randomized intervention trial of unstable angina. Lancet 2002;360:743-51.

4 Spacek R, Widimsky P, Straka Z, et al. Value of first day angiography/ angioplasty in evolving non-ST segment elevation myocardial infarction: an open multicenter randomized trial. The VINO study. Eur Heart J 2002;23:230-8.

5 Lee PY, Alexander KP, Hammill BG, et al. Representation of elderly persons and women in published randomized trials of acute coronary syndromes. JAMA 2001;286:708-13.

6 De Servi S, Cavallini C, Dellavalle A, et al. Non-ST-elevation acute coronary syndrome in the elderly: treatment strategies and 30-day outcome. Am Heart J 2004; 147:830-6.

7 Bach RG, Cannon CP, Weintraub WS, et al. The effect of routine, early invasive management on outcome for elderly patients with non-ST-segment elevation acute coronary syndromes. Ann Intern Med 2004;141:186-95.

8 Roques F, Michel P, Goldstone AR, et al. The logistic EuroSCORE. Eur Heart J 2003;24:881-2.

9 Califf RM, Abdelmeguid AE, Kuntz RE, et al. Myonecrosis after revascularization procedures. J Am Coll Cardiol 1998;31:241-51.

10 Katan MB. [Definition and treatment of hypercholesterolemia; consequences of the lipid research clinics study]. Ned Tiidschr Geneeskd 1984;128:1102-4.

11 Grundy SM, Pasternak R, Greenland P, et al. Assessment of cardiovascular risk by use of multiple-risk-factor assessment equations: a statement for healthcare professionals from the American Heart Association and the American College of Cardiology. Circulation 1999;100:1481-92.

12 Lloyd-Jones DM, Nam BH, D'Agostino RB Sr, et al. Parental cardiovascular disease as a risk factor for cardiovascular disease in middle-aged adults: a prospective study of parents and offspring. JAMA 2004;291:2204-11.

13 Shahi CN, Rathore SS, Wang Y, et al. Quality of care among elderly patients hospitalized with unstable angina. Am Heart J 2001;142:263-70.

14 Stone PH, Thompson B, Anderson HV, et al. Influence of race, sex, and age on management of unstable angina and non-Q-wave myocardial infarction: The TIMI III registry. JAMA 1996;275:1104-12.

15 Edwards FH, Clark RE, Schwartz M. Coronary artery bypass grafting: the Society of Thoracic Surgeons National Database experience. Ann Thorac Surg 1994;57:12-9.

16 Gersh BJ, Kronmal RA, Schaff HV, et al. Long-term (5 year) results of coronary bypass surgery in patients 65 years old or older: a report from the coronary artery surgery study. Circulation 1983;68:11190-9.

17 Rahimtoola SH, Grunkemeier GL, Starr A. Ten year survival after coronary artery bypass surgery for angina in patients aged 65 years and older. Circulation 1986:74:509-17.

18 Morrison DA, Bies RD, Sacks J. Coronary angioplasty for elderly patients with "high risk" unstable angina: short-term outcomes and long-term survival. J Am Coll Cardiol 1997;29:339-44.

19 Munoz JC, Alonso JJ, Duran JM, et al. Coronary stent implantation in patients older than 75 years of age: clinical profile and initial and long-term ( 3 years) outcome. Am Heart J 2002; 143:620-6.

20 Halon DA, Adawi S, Dobrecky-Mery l, et al. Importance of increasing age on the presentation and outcome of acute coronary syndromes in elderly patients. J Am Coll Cardiol 2004;43:346-52.

21 Craver JM, Puskas JD, Weintraub WW, et al. 601 octogenarians undergoing cardiac surgery: outcome and comparison with younger age groups. Ann Thorac Surg 1999;67:1104-10.

22 Alexander KP, Anstrom KJ, Muhlbaier LH, et al. Outcomes of cardiac surgery in patients $>$ or $=80$ years: results from the National Cardiovascular Network. J Am Coll Cardiol 2000;35:731-8.

23 Morrow DA. Troponins in patients with acute coronary syndromes: biologic, diagnostic, and therapeutic implications. Cardiovasc Toxicol 2001;1:105-10.

24 Morrow DA, Antman EM, Giugliano RP, et al. A simple risk index for rapid initial triage of patients with ST-elevation myocardial infarction: an InTIME II substudy. Lancet $2001 ; 358: 1571-5$. 